\title{
Rapid reconstruction of electric potentials over an incoherent scatter radar field-of-view
}

\author{
Valeriy G. Petrov*, Vladimir O. Papitashvili**, and C. Robert Clauer \\ Space Physics Research Laboratory, University of Michigan, Ann Arbor, Michigan, U.S.A.
}

(Received April 28, 2003; Revised September 2, 2003; Accepted September 2, 2003)

\begin{abstract}
A new technique is proposed for the rapid reconstruction of ionospheric electric fields from plasma drift measurements made by the Sondrestrøm incoherent scatter radar. We utilized the adaptive distribution of electric charges over and beyond of the radar field-of-view using a well-known method of regularization developed for solving the ill-posed problems. In this approach, no a priori assumptions are required concerning the regime of tangential components in the plasma drifts (i.e., radial electric fields). The test model calculations and comparisons of the reconstructed electric fields with ground-based geomagnetic field variations, global ionospheric convection modeling, and direct satellite observations demonstrate good results and, therefore, usefulness of the developed technique. This technique could be implemented as a tool for operational monitoring of the overall ionospheric convection dynamics within the incoherent (or coherent) radars field-of-view.
\end{abstract}

Key words: Geomagnetic data, ionospheric potentials, electric fields.

\section{Introduction}

The polar regions are known to be an ideal location for investigations of the solar wind and interplanetary magnetic field (IMF) interaction with the Earth's magnetosphere. It has been shown in many studies that the ionospheric plasma convection, electric fields, and field-aligned currents observed at high latitudes strongly depend on changes in the IMF strength and direction. Variations of the near-Earth interplanetary parameters cause almost instant changes in the dayside ionospheric convection patterns, which can be sensed by radar observations. The Sondrestrøm incoherent scatter radar (ISR) is located at $73.2^{\circ}$ of corrected geomagnetic (CGM) latitude, almost in the middle of the Greenland West Coast magnetometer chain (e.g., Wickwar et al., 1984; Clauer et al., 1995). At this location, the ISR observations provide a unique opportunity in studying electrodynamics of the polar ionosphere near the boundary between the auroral zone and polar cap. The dayside magnetic field lines emerging within the radar field-of-view (FOV) map to the vicinity of the dayside magnetopause, where the solar wind and IMF first encounter the Earth's magnetosphere.

The incoherent scatter radar measures a line-of-sight (LOS) component of the plasma convection velocity $\left(\mathbf{V}_{\mathbf{L O S}}\right)$ in the ionospheric $F$-region. Supposing that the plasma flow has no a component along the geomagnetic field $\mathbf{B}$ (or at least this component is significantly less than the plasma velocities perpendicular to B) and the flow is following only the $\mathbf{E} \times \mathbf{B}$ drift, one can calculate an electric field tangential component, perpendicular to the radar beam direction

\footnotetext{
*Also at IZMIRAN, Troitsk, Moscow Region, Russia

**A Also at National Science Foundation, Arlington, Virginia, U.S.A.
}

Copy right (C) The Society of Geomagnetism and Earth, Planetary and Space Sciences (SGEPSS); The Seismological Society of Japan; The Volcanological Society of Japan; The Geodetic Society of Japan; The Japanese Society for Planetary Sciences.
$\mathbf{E}_{\theta}=-\mathbf{V}_{\mathbf{L O S}} \times \mathbf{B}$. The electric field component along the radar beam is referred as a radial component $\left(E_{r}\right)$, but it is not directly measured. Although the Sondrestrøm radar can operate in various observation modes, in this study we used the data obtained in an azimuth scan mode. In this mode, the radar beam is usually elevated at $30^{\circ}$ angle above the horizon and it scans an entire range of geomagnetic azimuths from $0^{\circ}$ to $360^{\circ}$. Therefore, reliable velocity measurements are available within the range (along the Earth's surface) from 250 to $600 \mathrm{~km}$, with a spatial resolution of about $20 \mathrm{~km}$; the azimuth resolution is about $10^{\circ}$. Since the incoherent scatter radar measures only the LOS components of the $F$ region ion drift velocities, a technique is needed that would allow inferring an ionospheric electric field distributions in the radar FOV, thus obtaining realistic patterns of the ionospheric plasma convection. In this technique, we need to develop a method for deriving the second component of observed ion drifts, perpendicular to the radar beam. However, this problem cannot be solved without some additional assumptions.

The most common approach, used in the past for different radar studies (e.g., Doupnic et al., 1972; Evans, 1972; Behnke and Harper, 1973; Clauer and Banks, 1986), assumes that the ion drift velocity does not change (at least significantly) between two consecutive measurements located at the same range but along different azimuth scans. If $\mathbf{l}_{\mathbf{1}}$ and $\mathbf{l}_{2}$ are the unit vectors in the antenna azimuthal direction for the two radar beam positions and $\mathbf{V}=\left(V_{\text {east }}, V_{\text {north }}\right)$, is the plasma velocity vector, uniform over the two measurements $\mathrm{p} 1$ and $\mathrm{p} 2$, then the magnitudes of measured LOS velocities $V_{1 r}$ and $V_{2 r}$ at the respective points are the following: $V_{1 r}=\mathbf{l}_{\mathbf{1}} \cdot \mathbf{V}$ and $V_{2 r}=\mathbf{l}_{\mathbf{2}} \cdot \mathbf{V}$.

Solving these equations, one can obtain $V_{\text {east }}$ and $V_{\text {north }}$, and then the total velocity vector. Subsequently, the ionospheric electric fields can be derived from $\mathbf{E}=-\mathbf{V} \times \mathbf{B}$, 
where the (directed along the field line) vector $\mathbf{B}$ is taken from the International Geomagnetic Reference Field (IGRF) model (e.g., Mandea and Macmillan, 2000). However, if the observed ionospheric plasma flow is non-uniform, then these calculations could be wrong - a velocity component perpendicular to the average beam position may significantly be overestimated and the component along the beam may be underestimated. For example, Freeman et al. (1991) thoroughly addressed all possible errors in determination of the ionospheric electric fields with the single radar measurements.

Holt et al. (1984) and Foster et al. (1985) suggested a technique where the electric field was assumed irrotational in the radar FOV and, therefore, could be described by the electric potential distribution $\mathbf{E}=-\nabla \Phi$. They also assumed that this distribution does not change much during the entire radar scan $\left(360^{\circ}\right.$ or less). However, even if the potential distribution changes between two successive scans of the same point, the authors suggested interpolating the LOS velocities to some fixed universal time (UT). Therefore, having the electric field azimuthal component (derived from measured LOS velocities) in a closely spaced grid and taking into account $\nabla \times \mathbf{E}=\mathbf{0}$, one can calculate derivatives $\delta E_{r} / \delta \theta$ from $\delta E_{\theta} / \delta r$. Thus, if $E_{r}$ is known at any arbitrary fixed azimuth, then we can determine the electric fields at all other azimuths by integrating $\delta E_{r} / \delta \theta$.

Unfortunately, there is an uncertainty in determining the initial values for integration. Holt et al. (1984) suggested that $E_{r}$ could be equal to zero along a certain azimuth, but that requires an additional assumption where this azimuth $\left(E_{r}=0\right)$ should be placed within the radar azimuth scan. Foster et al. (1985) modified that requirement suggesting that the average value of $E_{r}$ along the entire radar scan should equal to zero for each range; Clauer et al. (1995) utilized this approach successfully in their study. Nevertheless, the latter approach is only valid for the homogeneous or special electric field distributions; this assumption becomes invalid if the convection rotation or velocity shears are observed within the radar FOV.

\section{Method}

In this study, we suggest a new approach for derivation of electric fields from the ISR measurements of ion drifts. As in previous studies, we suppose that the electric fields do not change significantly (i.e., they are stable) during an entire radar scan. Alternatively, we may suppose that the changes are linear and not too significant; then the FOV electric fields can be interpolated to some intermediate, fixed time. With the Sondrestrøm radar, the typical radar scan takes 4-6 minutes; therefore, the assumption on the field stability is often valid. However, this assumption may break for the transient events and time-varying phenomena as, for example, traveling convection vortices or pulsating electric fields. We also assume that the observed electric fields are of potential nature and this allows us reconstructing the FOV electric fields by an arbitrary distribution of electric charges $\rho=\operatorname{div} \mathbf{E}$. This approach does not require zeroing the electric potential elsewhere.

Assuming that the radar measurements are taken at the FOV grid-cell vertices and placing electric charges $Q_{i}=$ $J_{i} \cdot S_{i}$ in the center of each cell $i$ (where $J_{i}$ is the charge density and $S_{i}$ is the cell's area), we can calculate the electric fields generated by $Q_{i}$ at any arbitrary observation point $k$. Therefore, the total electric field generated by all charges at $k$ can be written as:

$$
\overline{E_{k}}=\sum_{i}^{N_{q}} J_{i} S_{i} \frac{\overline{r_{i k}}}{r_{i k}^{2}}
$$

where $\overline{r_{i k}}$ is the vector from the point $k$ to the point $i$ and $N_{q}$ is the number of charges. Here we understand that there are no static free charges in the conducting ionosphere and only field-aligned currents supply the charges. Therefore, our practical distribution of the charges actually describes the distribution of field-aligned currents that flow in and out of the two-dimensional ionosphere. As the geomagnetic field lines are equipotential, the charges must be evenly distributed along the field lines. This reduces the problem under investigation to a 2-D representation and in the equation above $E$ becomes dependent on distance as $1 / r$ rather than $1 / r^{2}$.

The radar LOS velocity observations provide tangential electric fields at each observation point. If we set the number of charges $N_{q}$ equal to the number of observation points $N_{p}$, then we can set $N$ equations with $N$ unknown variables; this seems to be enough for obtaining a solution of the equation system. However, this simple approach will not work because we also must place additional charges outside of the observation region; these charges are needed for describing correctly all possible electric field patterns. Moreover, a given distribution of the tangential electric fields can generally be modeled by a number of different charge distributions. For example, if we place an additional charge exactly at the radar location, then this charge will be producing only the radial electric fields everywhere over the radar FOV. Evidently, the latter does not affect the magnitudes of tangential electric components at the observation points, so the overall solution of the problem (that is, finding such distribution of the electric charges that produce the given distribution of tangential electric fields) is not unique in principal.

We suggest applying an additional limitation for resolving the problem: a total electric charge (or field-aligned current) must be minimal, satisfying a given distribution of tangential electric fields. Therefore, we should find such distribution of charges that minimizes the following expression:

$$
\sigma=\sum_{k=1}^{N_{p}} p_{k}\left(E_{k \theta q}-E_{k \theta m}\right)^{2}+\beta \sum_{i=1}^{N_{g}} Q_{i}^{2}
$$

Here $E_{k \theta q}$ is a tangential component of the electric field at the $k$-th point generated by all charges; $E_{k \theta m}$ is a measured tangential component of the electric field at the same point; $p_{k}$ is a weight coefficient determined by the measurement error; $Q_{i}$ is a charge in the $i$-th cell; and $\beta$ is a coefficient used for regularization of the minimization expression (2).

In our technique, we utilize a regularization method developed by Tikhonov and Arsenin (1977); it is often used for solution of so-called "ill-posed" problems - the problems that have no unique solution. The main idea of this method is to introduce some a priori condition and then to start looking for the solution which most effectively satisfies both the original equation and the introduced additional condition. In our 


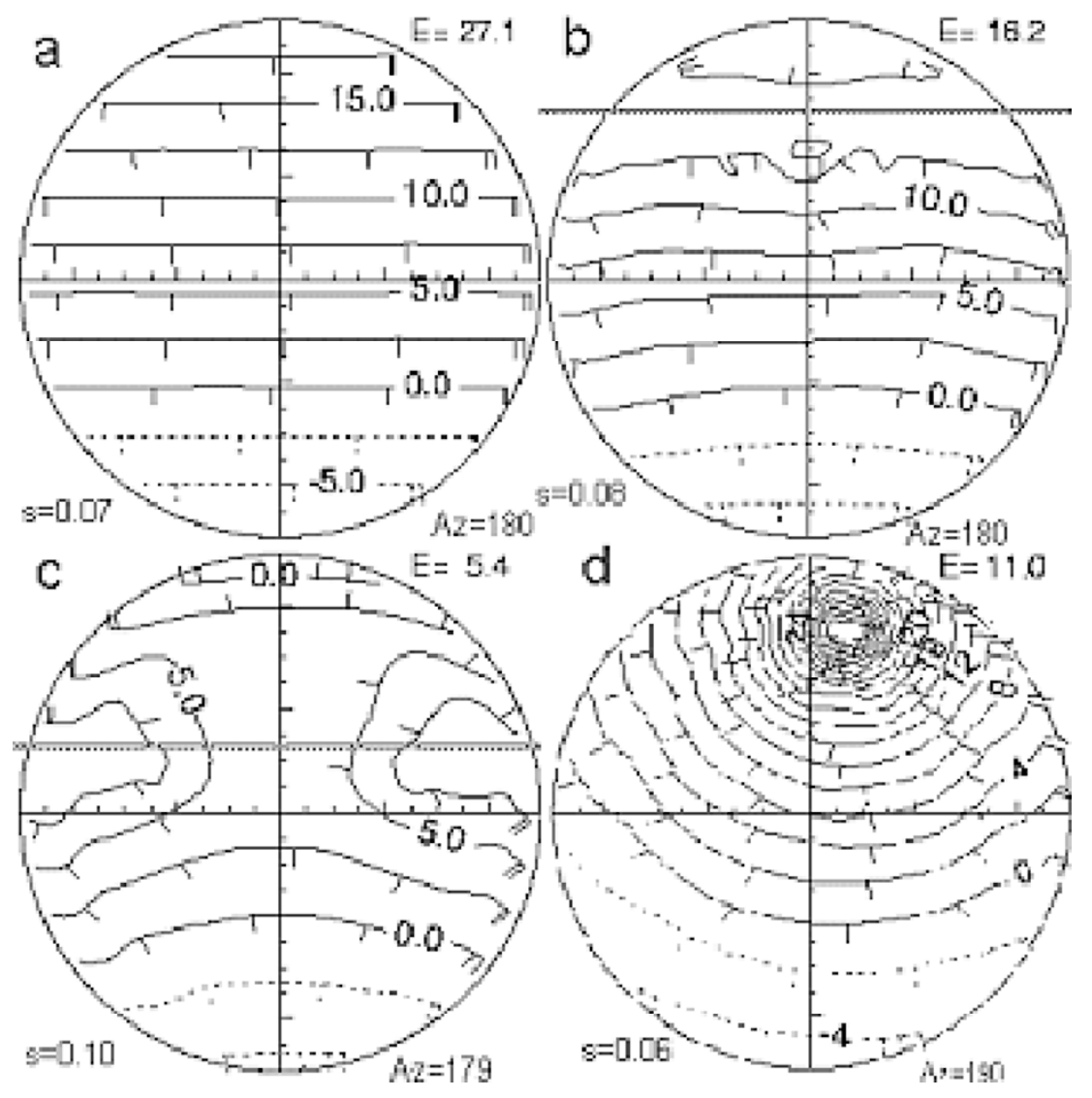

Fig. 1. Reconstruction of the electric potentials from modeled radar LOS ionospheric plasma convection velocities for: (a) a uniform west-east flow, (b), (c) velocity shear flows, and (d) a circular vortex flow. Magnetic north is on the top, east - to the right. $E$ is an average (over FOV) magnitude of the reconstructed electric fields; $A z$ is an average azimuth of the reconstructed electric field vectors, $s$ - a relative fitting error.

case, such condition is the minimum of the charges' power. Using (1), we can rewrite (2) as:

$$
\sigma=\sum_{k=1}^{N_{p}} p_{k}\left(\sum_{i=1}^{N_{q}} Q_{i} \frac{a_{i k}}{r_{i k}}-E_{k \theta m}\right)^{2}+\beta \sum_{i=1}^{N_{g}} Q_{i}^{2}
$$

where $a_{i k}$ is a cosine of the angle between $\overline{r_{i k}}$ and the direction of tangential component at the point $k$. Taking partial derivatives on $Q_{i}$ and setting them to zero, we obtain the system of $N_{q}$ equations with $N_{q}$ unknown variables:

$$
\left|\sum_{i=1}^{N_{q}}\left(\sum_{k=1}^{N_{p}} p_{k} \frac{a_{i k} a_{n k}}{r_{i k} r_{n k}}\right) Q_{i}+\beta Q_{n}=\sum_{k=1}^{N_{p}} p_{k} E_{k \theta m} \frac{a_{n k}}{r_{n k}}\right| n=1 \div N_{q}
$$

This system of equations can be solved by common numerical methods. However, the regularization coefficient $\beta$ should be determined experimentally, judging the model calculations by common-sense assumptions and experimental observations. For example, Popov et al. (2001) showed a number of examples suggesting that if $\beta$ is selected to be too small, then the solution becomes noisy and unstable; quite just the opposite, if $\beta$ is too large, then the solution cannot reproduce the original data accurately and fitting error becomes large. In our modeling, we tried different values of $\beta$ as well and concluded that the best results (stable solution, reproducing original data) for the Sondrestrøm ISR data can be obtained if $\beta=5 \cdot 10^{-5}$. In addition, our model calculations show that the obtained solutions are insensitive to the exact value of $\beta$ within the range $10^{-4}<\beta<10^{-5}$. We note that $\beta$ might be different if the distribution of observation points or the number of charges are different; so the only way to find proper value of $\beta$ is a "trial-and-error" method using known model calculations.

Figure 1 shows a few examples of the electric potential distributions reconstructed from the modeled (i.e., not observed) ionospheric plasma convection within the radar FOV. In these plots, ticks are drawn perpendicularly to the potential contours in the direction of decreasing field. The original field distributions were slightly "contaminated" by a random noise of $10 \%$ of the modeled $E$-field amplitude, thus emulating the radar measurement errors. The quality of electric field reconstruction can be judged here by a relative fitting error $s$, determined as a ratio of the square root mean differences between the measured and reconstructed radial components of the electric field to the average electric field magnitude calculated over the entire radar FOV. In the plots, the $E$ and $A z$ are the magnitude and direction of the reconstructed electric fields, respectively. As one can see, if the modeled distribution is approximately uniform, then the obtained $E$ and $A z$ values can be interpreted as the "real field" values; however, for the non-uniform plasma flow over the radar FOV these numbers might be underestimated or even have no sense.

Figure 1(a) shows a reconstruction of the potential distribution with the given uniform southward electric field of a magnitude $E_{0}=27.5 \mathrm{mV} / \mathrm{m}$. As seen, the reconstructed 


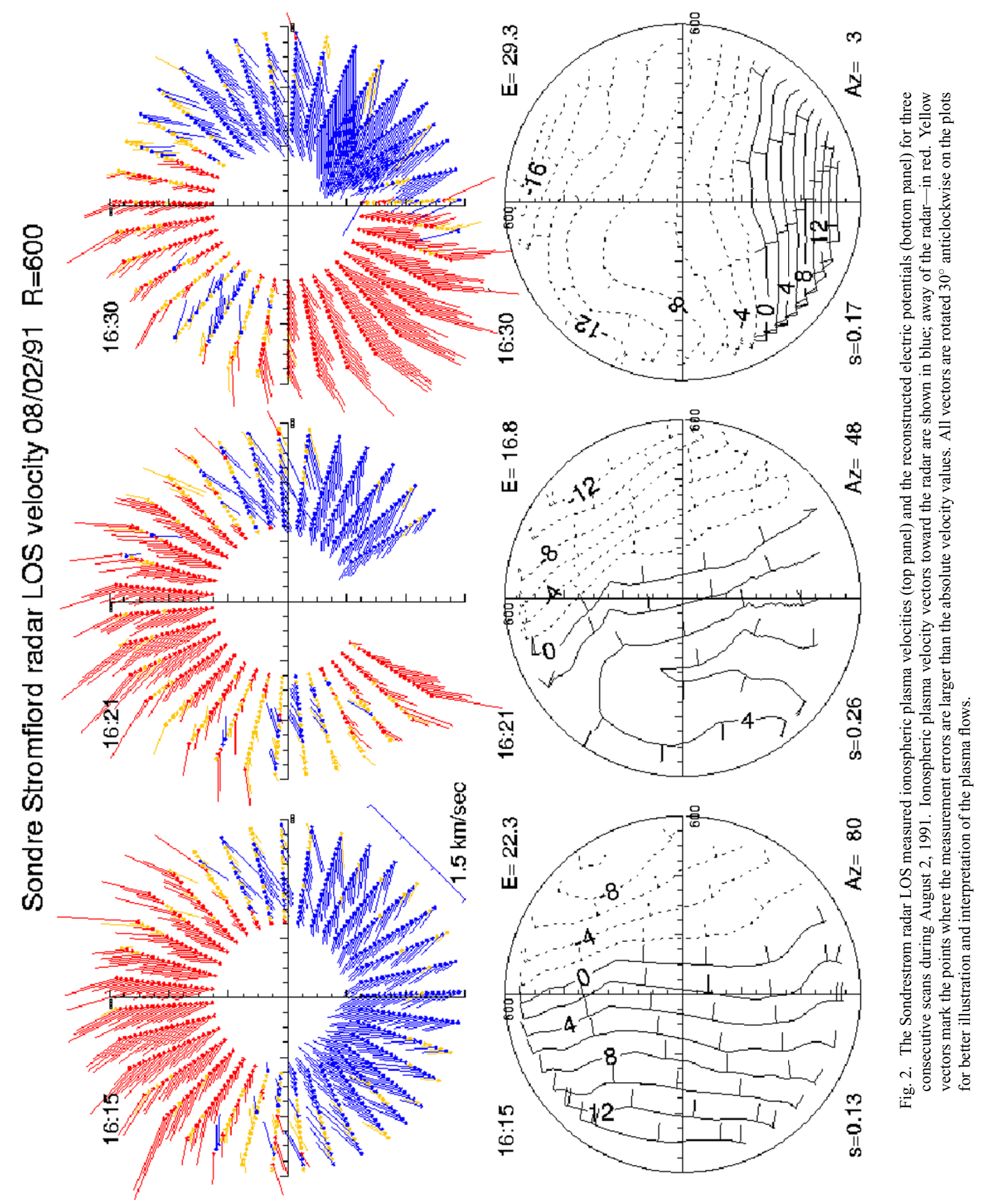


$E_{a v}=27.1 \mathrm{mV} / \mathrm{m}$ and $A z=180^{\circ}$ fit very well with the original distribution. Figure $1(\mathrm{~b})$ shows the reconstructed potentials with a velocity shear. Here the modeled, uniform southward electric field $27.5 \mathrm{mV} / \mathrm{m}$ fills an area from the southern edge of FOV up to $250 \mathrm{~km}$ north from a hypothetical radar; the northward electric field of the same magnitude is set farther north at $400 \mathrm{~km}$ with a linear transition near the reverse convection boundary (indicated by a thick horizontal line). As seen, this reconstruction fits with the originally modeled plasma convection as well.

Figure 1(c) shows another reconstruction similar to Fig. 1(b), but the reverse boundary is now located much closer $(125 \mathrm{~km})$ to the hypothetical radar. This reconstruction gives satisfactory location of the reverse boundary, but the reconstructed shear area is much wider because of the proximity to the hypothetical radar. The last Figure 1(d) shows reconstruction of the potential distribution for a localized source (i.e., a charge or a field-aligned current filament) located north of the radar, which induces radial electric fields and the circular plasma flow. As seen, the reconstruction of the source pattern is also reasonable, though the average $E$ field becomes irrelevant.

Thus, these modeling results show that we practically can obtain almost an exact solution of the problem for the uniform electric field distribution, even with the added noise. A relatively high fitting error $s \approx 0.06-0.10$ is mainly controlled by the added random noise; that is, our method may reconstruct the original, clean uniform field almost ideally, but we preferred to compare our reconstructions with "quasinoisy" data. In the case of a shear located far from the radar position, the method gives good results with the field magnitude of $\approx 60 \%$ of the original model; however, disturbed electric fields are seen over the reconstructed transition area. We note that relatively high disturbances in the transition area can be generated by the selected "quasi-noise" level (10\% of $E_{0}$ ) because the noise of the same magnitude was added to all points over the FOV. Therefore, where the original electric fields were smaller (e.g., in the transition area), then the noise-to-signal ratio was much higher. On the other hand, in attempting the reconstruction of the shear boundary, we have to add an infinite line of charges, which is certainly limited by the FOV boundary; thus, limiting the technique's capabilities.

Figure 1(b) and Figure 1(c) show that the reconstructed patterns are generally near-uniform (as the original field distribution) southward of the shear boundary; the technique is even able to reconstruct the northward fields well beyond the shear area. At the same time, when the shear boundary is located closer to the radar (Fig. 1(c)), some significant distortions (from the original model) are seen in the shear area.

In practice, the Sondrestrøm ISR does not receive return signals from the distances up to $\sim 250 \mathrm{~km}$ during the horizontal scans with the beam elevation of $30^{\circ}$; thus there are no observations of plasma flows within this range. In our modeling, we simulated the "lack" of near-radar data for Fig. 1 as well; this suggests that the significant field distortions within the shear region close to the radar might be caused by the absence of measurements. We can improve our reconstruction pattern over the shear boundary by placing additional charges in the central part of the radar FOV. This increases the second term in Eq. (2) but does not change much the first term because there are no measured data in this area. However, that might make minimization of $\sigma$ worse; therefore, the equation solver does not place additional charges over the FOV central part. It cannot be considered as a deficiency of the proposed method because there no radar measurements are made within that region anyway and we cannot expect the correct solution here. However, we must be very careful in interpreting obtained rotational discontinuities in the region without measurements.

\section{Method Evaluation}

As noted above, the reconstruction of electric potentials from ISR's data is the ill-posed problem, which generates an infinite set of all possible solutions. Therefore, to be on a sure ground, we should compare the reconstructed potential patterns with other available observations.

The upper panels in Figs. 2 and 3 show a few examples of the radar LOS velocity distributions, with the "away-fromradar" velocities in red, and the "toward-radar" velocities in blue. Yellow vectors mark the points where the measurement errors are larger than the absolute ion velocity values. All these vectors are rotated anticlockwise by $30^{\circ}$ from the radial direction to make the vectors visible for interpretation; however, the reader should keep in mind that this rotation is performed only for illustration purposes and the real direction of these vectors is purely radial.

Ridley and Clauer (1996) extensively investigated similar LOS velocity distributions and showed that in the case of a uniform flow the radar clearly observes a region with the flow toward the radar and another region with the flow away from the radar. In the case of shear flows, the radar observes four different LOS velocity regions: two regions with the towardradar flows and two regions with the away flows. Thus, the LOS velocity plots for 1615 UT of August 2, 1991, and for 1706 UT of October 7, 2000, are the uniform flows; shear flows are observed during other time intervals shown in these figures.

The bottom panels in Figs. 2 and 3 show the reconstructed potentials over the radar FOV, calculated from the measured LOS velocity distributions by utilizing our new technique. As seen, the reconstructed fields at 1615 UT and 1630 UT are in good agreement with the original observations $(s \leq$ 0.17). For example, at 1615 UT the northward plasma flow shows higher velocities in the northern part of the radar FOV; the reconstructed electric fields are also larger here. The potential plot for 1621 UT gives about the same distribution as for 1615 UT at the eastern part of the radar FOV, but the LOS velocity vectors point to the opposite direction at the western part of the radar FOV; thus our fitting here is less optimal $(s=0.26)$. We guess that for this case electric fields changed during the radar scan and our main assumption on the stationary electric field became invalid. However, in general the reconstructed pattern for 1621 UT fits well with the LOS velocity observations. At $1630 \mathrm{UT}$, the westward flow is much faster at the eastern and southern portions of the FOV; all these features are quite evident in the reconstructed potential distribution.

Evaluating our new method, we also compared time series of the (averaged over the radar FOV) electric field 

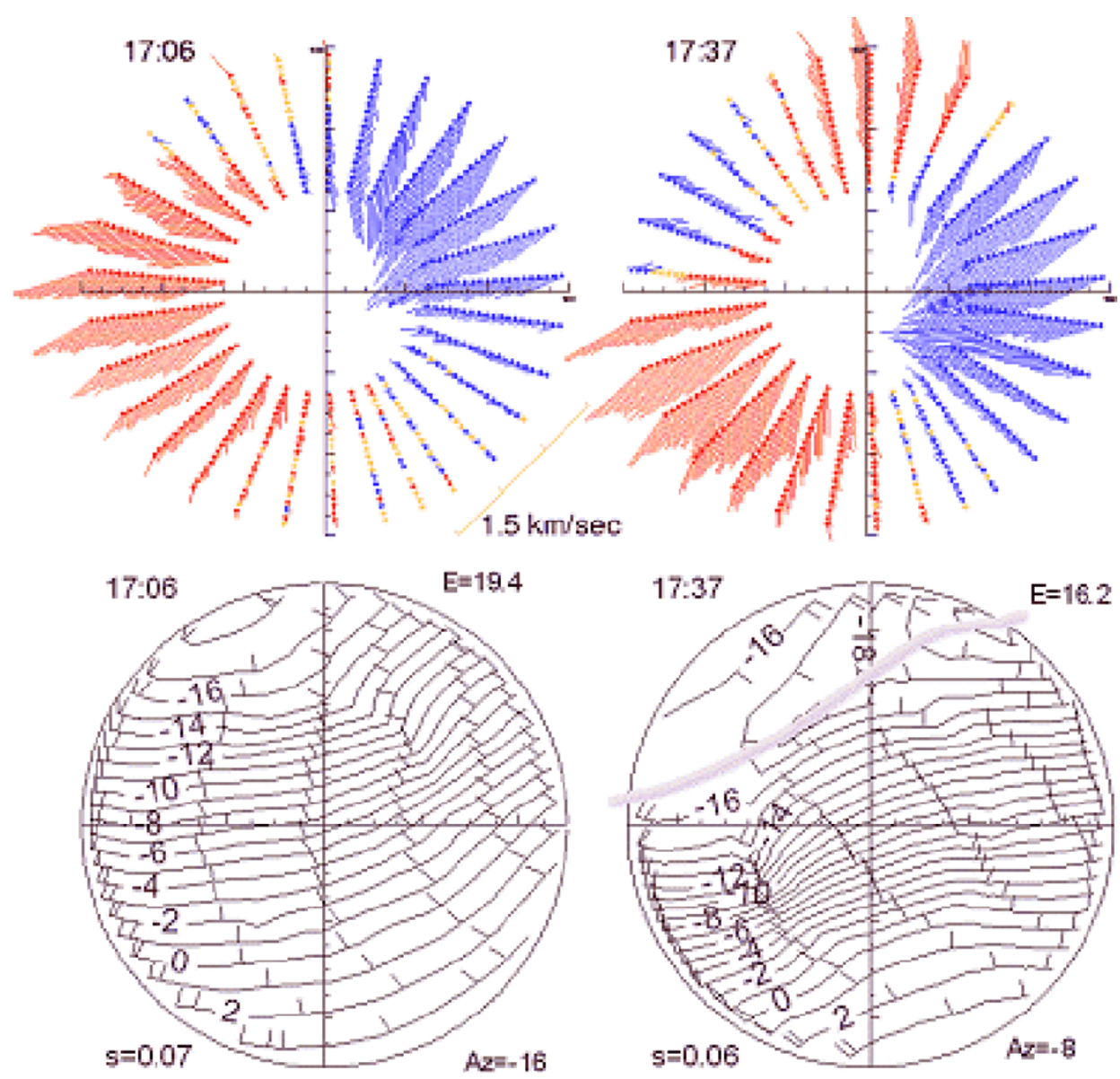

Fig. 3. Same as on Fig. 2 but for two ISR scans during October 7, 2000.

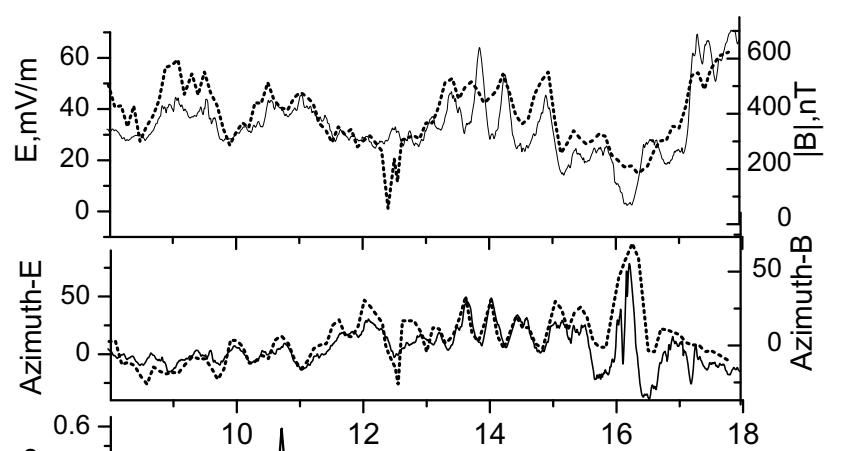

(12 14
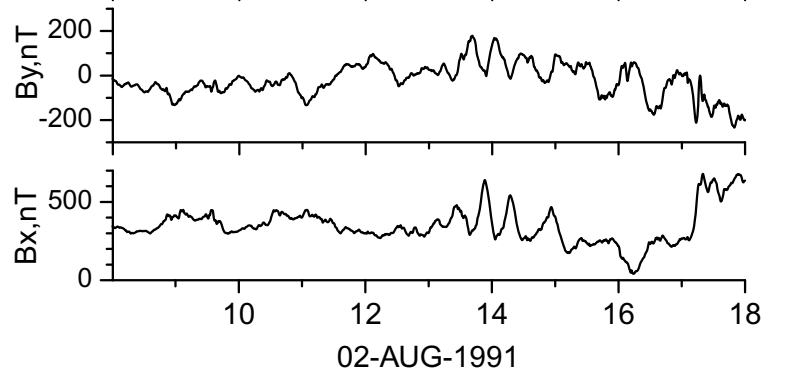

Fig. 4. Measured magnetic variations (solid lines) and reconstructed electric fields (dotted lines) from the Sondrestrøm radar observations during August 2, 1991. and its direction (azimuth) with the geomagnetic variations recorded on the ground. According to the well-known theorem (Fukushima, 1976), only ionospheric Hall currents control ground geomagnetic variations in the polar regions because the magnetic effect of ionospheric Pedersen currents is canceled right below the ionosphere by the magnetic effect of field-aligned currents (in the assumption that the field lines are near-vertical). Therefore, the direction of a ground magnetic field perturbation vector should be parallel to the direction of the corresponding ionospheric electric field and the magnetic variation magnitude - proportional to the ionospheric current intensity. However, if the ionospheric Hall conductivity is uniform (i.e., no significant conductivity gradients are expected in the vicinity of observations), then the magnetic field disturbance magnitude will be also proportional to the ionospheric electric field magnitude. Certainly, the correlation between these parameters cannot be $100 \%$ because the conductivities vary and the ground magnetic field variations can also be (at least partially) affected by some other sources; for example, by the remote ionospheric and field-aligned currents.

Figure 4 shows variations of the average electric field (magnitude E and direction Azimuth-E, plotted as dotted lines; two upper graphs) for the entire radar experiment interval on August 2, 1991 (0900-1800 UT) studied by Clauer et al. (1995).

The middle panel shows fitting errors, where one can see 
that the errors were less than 0.1 about a half of time; the error exceeds 0.2 only in a few scans. The reconstructed electric fields point generally in the northward direction deviating from north by $-20 /+80$ degrees. Unfortunately, the Sondrestrøm magnetometer data were unavailable for the interval under the study and we used magnetometer data from the station Attu, located $\sim 150 \mathrm{~km}$ northward of the Sondrestrøm radar. Two lower graphs in Fig. 4 show variations of the $B_{X}$ and $B_{Y}$ geomagnetic field components (as deviations from an adopted quiet level) at Attu; the total horizontal magnetic field $|B|=\sqrt{B_{X}^{2}+B_{Y}^{2}}$ and its vectors' azimuths are shown as solid lines in the two upper graphs, plotted together with the dotted lines representing the reconstructed electric field parameters.

Figure 5 shows similar graphs for the radar experiment on October 7, 2000, where we used the Sondrestrøm magnetometer data for the comparison. One can see here a good agreement between the magnetic and electric field time series until $\sim 1630$ UT. In this case, the estimated Hall conductivity is about two times lower than in the previous case because these observations are taken during equinox and the magnetic activity was particularly low. After 1630 UT, the observed magnetic fields became significantly more disturbed; this could be caused either by the enhanced ionospheric conductivity within the radar FOV (for example, from the localized particle precipitation) or by more complex original electric field distributions in the radar FOV than we assumed for the reconstruction.

Figure 3 shows the observed LOS velocities and reconstructed electric potential patterns for two time instances right after 1630 UT on October 7, 2000. At 1706 UT, the LOS velocity distribution shows that the drifts slow down with the distance from the radar in the northwest quadrant of the observed region, but in the northeast and southwest quadrants the LOS velocities are of the same value. This suggests that the plasma flow in the radar FOV was non-uniform and our reconstructed potentials confirm this fact: the distances between contour lines in the northwest quadrant are about five times wider and in the southeast quadrant they are about three times wider than in the other quadrants; therefore, the electric fields in northwest and southeast quadrants are weaker than elsewhere. The radar observed a convection reversal boundary at 1737 UT; the plasma flows southwestward in the central and southeastern quadrants, and the plasma flows northeastward in the northwestern FOV quadrant. The potential distributions also show the electric field reverse boundary, marked by a solid gray line in Fig. 3 .

We note that the electric fields plotted in Figs. 4 and 5 are the average fields calculated over the entire radar FOV; therefore, if the electric fields are non-uniform, the average value might underestimate significantly the real electric fields above the magnetometer station. Complex distributions of the electric fields can be a result of the particle precipitation that makes the local conductivity irregular.

Nevertheless, the comparison of the $|\mathrm{B}|$ and $\mathrm{E}$ time series as well as the corresponding vectors' azimuths in Fig. 5 shows that the correlation between the electric field and magnetic variations is good. In addition, considering the ionospheric current to be infinite and assuming that the ground

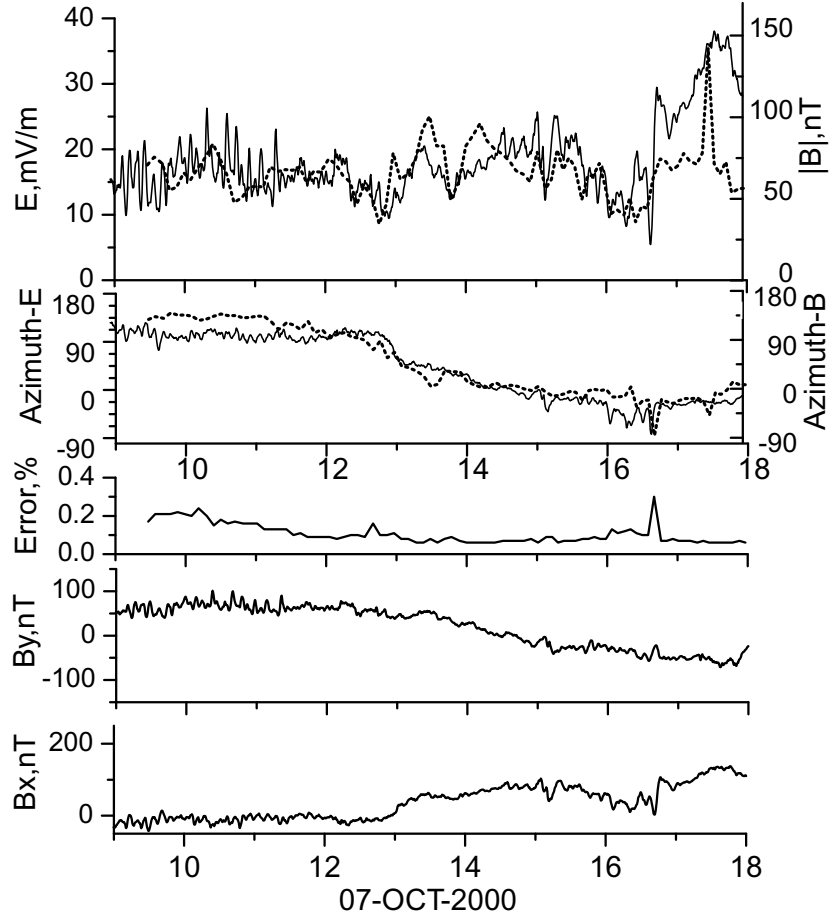

Fig. 5. Same as on Fig. 4 but for October 7, 2000

induction effect increases the observed magnetic horizontal vector by a factor of 1.5 (Porath et al., 1970), we can estimate the average Hall conductivity in the radar FOV from our electric and magnetic field data. From the data shown in Fig. 5, this conductivity amounts to 9-10 S, which agrees well with other available estimates of the ionospheric conductivity for the summer season (e.g., Hardy et al., 1987).

To further evaluate our new technique, we statistically compared the electric fields reconstructed from radar observations and the electric fields obtained from the global DMSP-based ionospheric convection model DICM (Papitashvili and Rich, 2002); see an interactive display at http://www.sprl.umich.edu/mist/limie.html as well. We processed all our radar experiments in 2000 (eight days in March and October, from 1000 UT to 1600 UT), selecting the time intervals when the IMF was stable during at least one hour (to exclude errors related to the uncertainty in the IMF propagation time delay) and the electric fields in the radar FOV were fairly uniform, so we can expect that electric field reconstruction will work well. Figure 6 shows the comparison of electric fields reconstructed from experimental radar data and modeled from DICM. The DICM is constructed from DMSP ion drift observations taken at the altitude of $\sim 850$ $\mathrm{km}$, while the radar data are derived for the average altitude of $\sim 200 \mathrm{~km}$; therefore, we mapped down the DICM-based electric fields taking into account geometry of geomagnetic field lines at high latitudes.

A solid line at the plot represents the linear fit to the compared data; the dotted lines indicate a $95 \%$ confidence limit of the fitting. As seen, the electric field amplitudes reconstructed from the radar data are slightly larger than the corresponding electric fields inferred from the DICM modeling; nevertheless, the comparison looks satisfactory. Significant scattering of experimental data is common when the statisti- 


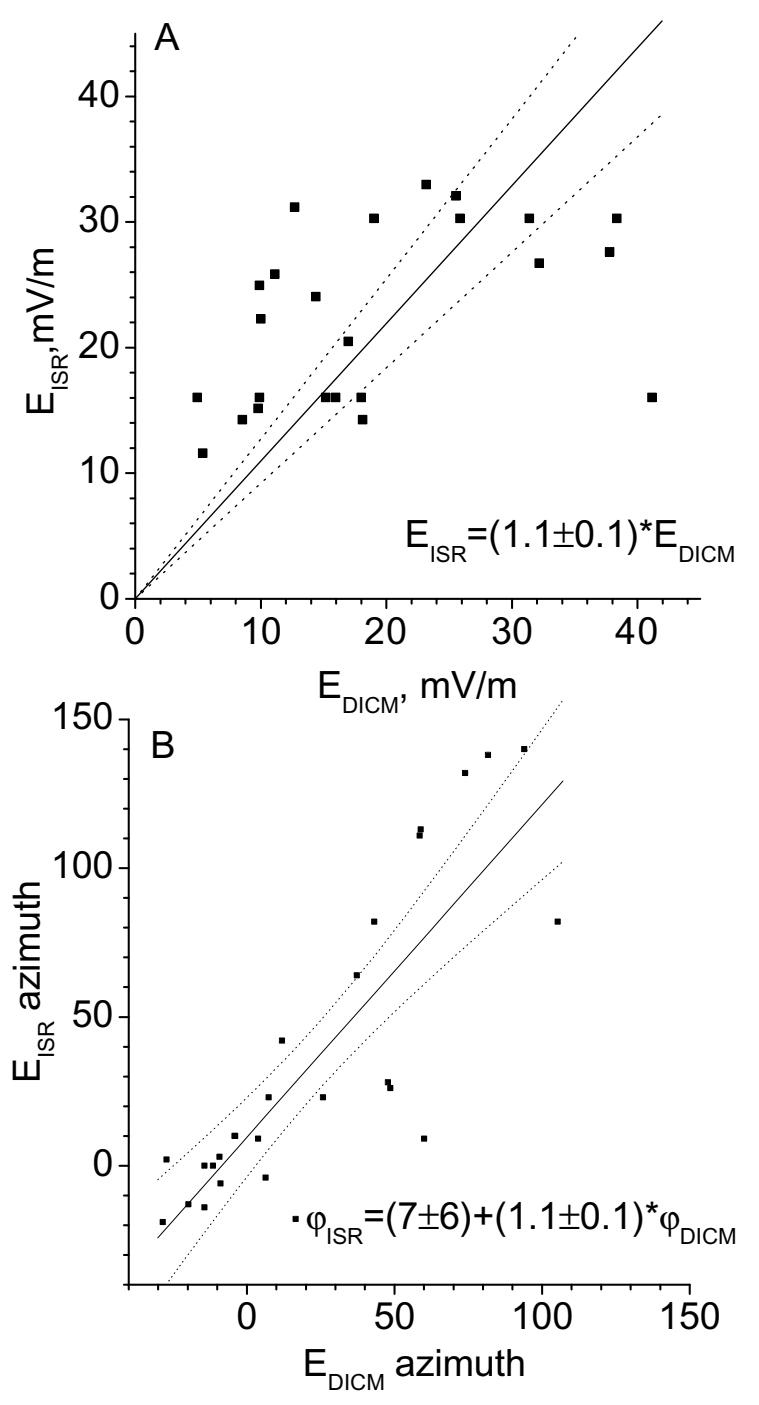

Fig. 6. Comparisons of the reconstructed electric fields over the Sondrestrøm radar FOV and the electric fields inferred from the DMSP Ionospheric Convection Model (DICM). The latter fields are mapped down to the average radar measurements altitude of $\sim 200 \mathrm{~km}$.

cal model is compared with the case-study events. Statistical estimations show that the obtained relations are statistically reliable with confidence more than 0.9995 (the correlation coefficient $\mathrm{R}$ is 0.65 for $\mathrm{E}$ field amplitude and 0.85 for azimuth) and the global model and our reconstructions capture approximately the same electric fields in the daytime sector.

However, our evaluation will not be complete if we do not compare the reconstructed electric fields with direct satellite observations. For this purpose, we looked for good DMSP passes over the radar FOV in the recent radar experiments. Unfortunately, there were no passes available where the satellite would be crossing the radar FOV nearperpendicularly to the convection flow. Figure 7 shows one of the examples for October 29, 2000, when the DMSP satellite F-15 crossed the radar FOV in less than 2 minutes. The top panel shows the reconstructed potential pattern from the radar plasma flow observations during the scan at 14521458 UT and the overlapping satellite track for 1452-1454 UT (entering from north). A solid line at the bottom plot shows a measured profile of the "along-the-track" electric

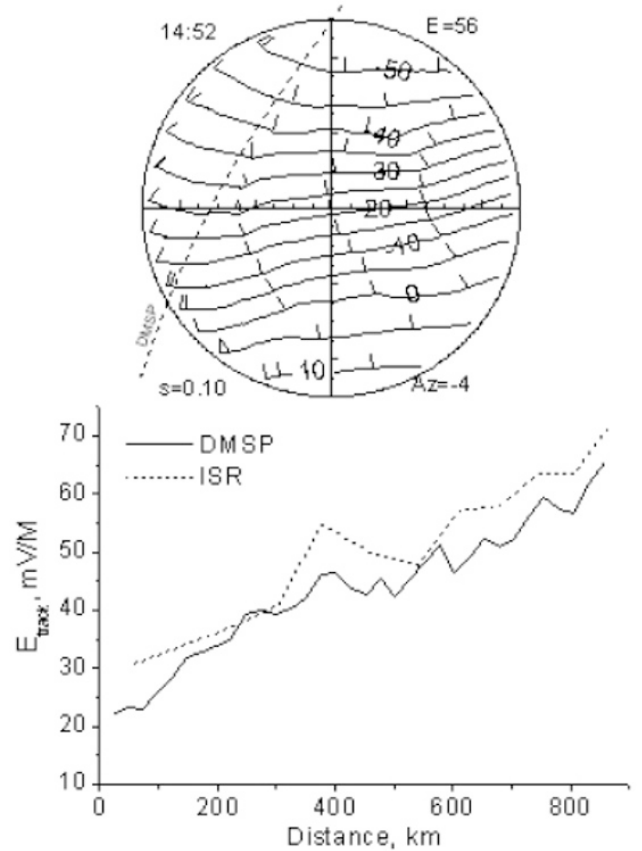

Fig. 7. Comparison of the reconstructed electric potential pattern over the Sondrestrøm radar FOV (top panel) and the electric fields (bottom panel) inferred from the direct DMSP satellite observations of ion drifts (solid line) and calculated from the gradients of reconstructed potentials (dotted line). Both graphs begin from the satellite's northernmost entry into the radar field-of-view.

fields inferred from the cross-track ion drifts and mapped down to $\sim 200 \mathrm{~km}$ altitude. The dotted line on the same graph shows the "along-the-track" electric field component calculated from the gradients in the reconstructed potential distribution. As seen, the agreement between two graphs is good enough, showing that the reconstructed electric fields are $\sim 1.1$ times larger than the satellite data.

Thus, we can conclude that the average electric field amplitudes reconstructed from the radar observations and obtained either from direct DMSP measurements or from the DMSP-based ionospheric convection model under approximately time stationary and uniform electric field conditions are in agreement within the accuracy of our calculations. The scattering of observation (radar and satellite) data is mainly caused by local spatial-temporal variability in the measured (by either instrument) electric fields. Although the radar electric field measurements very often show non-uniform distributions displaying shears in the radar FOV, our technique (as well as the statistical convection model) may smear out some of this variability in producing the ionospheric potential patterns. Further validation of the proposed technique can be continued by building good statistics from multicase reconstructions of the time-varying phenomena when the plasma flow is highly irregular in the radar FOV. This effort is currently underway in our research group but it is certainly out of scope of this technical study.

\section{Conclusions}

In this study, we described and evaluated a new technique developed for the reconstruction of electric potential distributions in the radar field-of-view from the Sondrestrøm ISR 
observations of the radial (along the radar's beams) plasma velocities. As in the most methods of such kind, we suppose that electric field is potential and stationary at least during radar scan period, but our method does not require a priori assumptions concerning the regime of tangential components in the plasma drifts (that is, radial electric fields). The developed technique can be recommended for getting realistic values and distributions of the electric fields over the LOS velocity distribution pattern when there are grounds for supposing that general assumptions are valid.

We modeled a few typical plasma flows often seen in the radar FOV with the added noise and then reconstructed these distributions using the new technique; the results are satisfactory. Then we reconstructed electric fields from the Sondrestrøm radar observations for selected events and compared these results with the ground-based geomagnetic field variations, with the global convection patterns modeled by the DMSP-based ionospheric convection model, and with the direct DMSP observations of ionospheric electric fields inferred from cross-track ion drifts. These comparisons demonstrate the usefulness of our technique; however, more detailed investigations of the proposed method are needed for further validation of the technique.

Acknowledgments. The authors are grateful to John C. Foster and John M. Holt of MIT's Haystack Observatory for helpful discussions. The DMSP data were kindly provided by Frederick J. Rich of AFRL. We also thank both referees for helpful comments. This work was supported by the National Science Foundation awards OPP-9876473 and OPP-0220735 to the University of Michigan.

\section{References}

Behnke, R. A. and R. M. Harper, Vector measurement of F region ion transport at Arecibo, J. Geophys. Res.,78, 8222-8234, 1973.

Clauer, C. R. and P. M. Banks, Relationship of the interplanetary electric field to the high-latitude ionospheric electric field and currents: observations and model simulation, J. Geophys. Res., 91, 6959-6971, 1986.

Clauer, C. R., P. Stauning, T. J. Rosenberg, E. Friis-Christensen, P. M Miller, and R. J. Sitar, Observation of solar-wind-driven modulation of the dayside ionospheric DPY current system, J. Geophys. Res., 100, 7697-7713, 1995.
Doupnic, J. R., P. M. Banks, M. J. Baron, C. L. Rino, and J. Petriceks, Direct measurements of plasma drift velocities at high magnetic latitude, J. Geophys. Res., 77, 4268-4275, 1972.

Evans, J. V., Measurements of horizontal drifts in the $\mathrm{E}$ and $\mathrm{F}$ regions at Millstone Hill, J. Geophys. Res., 77, 2341-2350, 1972.

Foster, J. C., J. M. Holt, J. D. Kelly, and V. B. Wickwar, High-resolution observations of electric fields and F-region plasma parameters in the cleft ionosphere, in The Polar Cusp, Edited by J. A. Holtet and A. Egeland, NATO ASI Series, Reidel Publishing Co., pp. 349-364, Mass., 1985.

Freeman, M. P., J. M. Ruohoniemi, and R. A. Greenwald, The determination of time-stationary two dimensional convection patterns with singlestation radars, J. Geophys. Res., 96, 15735-15740, 1991.

Fukushima, N., Generalized theorem for no ground magnetic effect of vertical currents connected with Pedersen currents in the uniformconductivity ionosphere, Rep. Ionos. Space Res. Japan, 30, 35-40, 1976.

Hardy, D. A., M. S. Gussenhoven, R. Raistrick, and W. J. McNeil, Statistical and functional representations of the pattern of auroral energy flux, number flux, and conductivity, J. Geophys. Res., 92, 12,275-12,294, 1987.

Holt, J. M., R. H. Wang, and J. V. Evans, Millstone Hill measurements on 26 February 1979 during the solar eclipse and formation of a midday $\mathrm{F}$ region trough, J. Atmos. Terr. Phys., 46, 251-264, 1984.

Mandea, M. and S. Macmillan, International Geomagnetic Reference Field - the eighth generation, Earth Planets Space, 52, 1119-1124, 2000

Papitashvili, V. O. and F. J. Rich, High-latitude ionospheric convection models derived from Defense Meteorological Satellite Program ion drift observations and parameterized by the interplanetary magnetic field strength and direction, J. Geophys. Res., 107(A8), 10.1029/2001JA000264, 2002.

Popov, V. A., V. O. Papitashvili, and J. F. Watermann, Modeling of equivalent ionospheric currents from meridian magnetometer chain data, Earth Planets Space, 53(2), 129-137, 2001

Porath, H., D. W. Oldenburg, and D. I. Gough, Separation of magnetic variation fields and conductive structures in the western United States, Geophys. J. Roy. Astron. Soc., 19, 237-260, 1970.

Ridley, A. J. and C. R. Clauer, Characterization of the dynamical variations of the dayside high-latitude ionospheric convection reversal boundary and relationship to interplanetary magnetic field orientation, J. Geophys. Res., 104, 101, 10,919-10,938, 1996.

Tikhonov, A. N. and V. Ia. Arsenin, Solution of ill-posed problems, 258 pp., Halsted Press, New York, 1977.

Wickwar, V. B., J. D. Kelly, O. de la Beaujardiere, C. A. Leger, F. Steenstrup, and C. H. Dawson, A Sondrestrøm overview, Geophys. Res. Lett., 11, 883-866, 1984.

V. G. Petrov (e-mail: vpetrov@engin.umich.edu), V. O. Papitashvili (email: papita@umich.edu), and C. R. Clauer (e-mail: rclauer@umich.edu) 